\title{
Models at Play: Using Dynamic Field Theory to Understand Looking and Learning in Dyadic Interactions ${ }^{+}$
}

\author{
John P. Spencer \\ School of Psychology, University of East Anglia, Norwich NR4 7TJ, UK; j.spencer@uea.ac.uk \\ + Presented at the IS4SI 2017 Summit DIGITALISATION FOR A SUSTAINABLE SOCIETY, Gothenburg, \\ Sweden, 12-16 June 2017.
}

Published: 9 June 2017

\begin{abstract}
Although cognitive and social development are often studied in isolation, many researchers have demonstrated convincingly that cognition and the social environment are inseparable components of development. For instance, the social context plays a crucial role in many facets of cognitive development. Critically, the mechanisms by which social interactions impact cognitive development remain poorly understood. Here, we present a dynamic field model that elucidates the neural and behavioral mechanisms by which social interactions contribute to developmental changes in cognition and how these influences are reciprocal in nature.

The goal of our work is to understand the mechanisms by which parental responsiveness impacts the social and cognitive development of term and preterm infants. We present an autonomous dynamic field model that looks about its environment containing multiple virtual objects. This neural system encodes and forms memories for the objects being looked at and captures the looking and memory formation abilities of typically developing and preterm infants and adults. We present several simulation experiments in which a parent model and preterm infant model share the same virtual world. We illustrate that a simple Hebbian learning process within the neural and behavioral systems of the parent and infant models is responsible for changes in how the infant model performs in a memory task and how the models learn to interact with each other.
\end{abstract}

(C) 2017 by the author. Licensee MDPI, Basel, Switzerland. This article is an open access article distributed under the terms and conditions of the Creative Commons Attribution (CC BY) license (http://creativecommons.org/licenses/by/4.0/). 